\title{
Sequential blanking effects with matrix displays
}

\author{
WILLIAM W. AGRESTI \\ University of Michigan, Dearborn, Michigan 48128 \\ and \\ MARK S. MAYZNER \\ Loyola University, Chicago, Illinois 60626
}

\begin{abstract}
The present study demonstrates sequential blanking effects can easily be obtained with matrix-type displays as well as linear displays, as shown in earlier work.
\end{abstract}

Sequential blanking is a perceptual effect first reported 12 years ago as part of a continuing investigation of visual information processing mechanisms. The effect is clearly observed under the following conditions. A computer-driven cathode-ray tube (CRT) display system generates 10 characters, for example, SOMERSAULT, across the screen. The timing and ordering of the individual character presentations is critical to the production of sequential blanking. The presentation time ("on" time) of an individual character is $10 \mathrm{msec}$, with an interval of $10 \mathrm{msec}$ ("off" time) before the next presentation begins. The order of display of the 10 characters is $1,6,2,7,3,8,4,9,5,10$; that is, $S$ is displayed first, $M$ second, $R$ third, and so on. When such timing and ordering obtained, observers report seeing only five letters, namely, $\mathrm{O}, \mathrm{E}, \mathrm{S}, \mathrm{U}, \mathrm{T}$, with the other five letters missing.

This sequential blanking effect endures under some changes in ordering and input characters. For example, another display order that produces sequential blanking is $3,1,4,2,5,7,9,6,8,10$. The missing letters are those displayed $2,5,6,7,8$. The sensitivity of the effect to changing spatiotemporal conditions is further reported by Mayzner and Tresselt (1970).

Earlier studies of sequential blanking have focused on a linear array of stimuli presented across a display screen. The present experiment was conducted to test whether sequential blanking occurs when a twodimensional array of characters provides the input. Four different square matrices, consisting of letter Xs, were used: (1) 4 Xs arranged in a 2 by 2 matrix, (2) 9 Xs arranged 3 by 3 , (3) $25 \mathrm{Xs}$ arranged 5 by 5 , and (4) 49 Xs arranged 7 by 7 .

The interest in this experiment is in the number of Xs that subjects report actually seeing from the matrix. If the reported number is less than the number of Xs actually displayed, such evidence might indicate that sequential blanking has occurred.

This research was supported in part by National Science Foundation Grants BG22785 and BNS75-09800 A02 to M. S. Mayzner.

\section{METHOD}

The five students who volunteered for the experiment were undergraduate or graduate students. The stimuli were presented on a VR-14 CRT display driven by a PDP-8/e computer. The surface of the CRT was coated with an ultrashort-persistence (P24) phosphor. In Mayzner $(1968,1975)$, the laboratory is described in detail.

The stimuli consist of letter Xs arranged in square matrix. Display luminance was measured at $1 \mathrm{~mL}$, and the subject was seated $2 \mathrm{ft}$ in front of the display screen. Four matrix sizes were used, 2 by 2,3 by 3,5 by 5 , and 7 by 7 . For each size, two types of ordering were used. In the first (nonrandom), the Xs in the matrix were presented in order sequentially from left to right beginning with the top row and continuing down to the bottom row. The second ordering was random; that is, there was no pattern to the order in which the individual Xs in the matrix were displayed. The sequence was determined by a randomnumber generator that is part of the computer program that controlled the display. The same order was not used repeatedly, but rather, a new sequence was generated for each trial. For each matrix size, a subject was given 10 trials of the nonrandom order and 50 trials of the random order. Further, the 60 trials were randomized over ordering, thus interspersing the nonrandom trials with the random trials.

The timing of the displays was not constant over the four matrix types, because the number of Xs displayed varied so widely from 4 Xs in the 2 by 2 array to 49 Xs in the 7 by 7 array. Instead, the choice of on and off times was made by considering those times that have produced sequential blanking in linear arrays. In earlier studies (e.g., Mayzner \& Tresselt, 1970), the effect was most sharply observed when the total display time was approximately $200 \mathrm{msec}$, for input strings of 5 or 10 characters. For example, with five characters the on time was $20 \mathrm{msec}$ and the off time was $20 \mathrm{msec}$. With 10 characters, the on time and off time were each 10 msec.

The on time and off time in this experiment were chosen so that the total display was reasonably close to $200 \mathrm{msec}$, except for the 7 by 7 matrix. With $49 \mathrm{Xs}$ to be displayed, requiring a total display time of approximately $200 \mathrm{msec}$ would have reduced the individual letter presentation times so much that all 49 Xs would have appeared to the subject to be simultaneous. For this reason, the individual letter on times were kept at $10 \mathrm{msec}$. The display times are summarized in Table 1.

Each subject first received the set of 60 trials consisting of 2 by 2 arrays, then the 60 trials of 3 by 3 arrays, then 5 by 5 , and finally, 7 by 7 , for a total of 240 displays. Before each of the four sets of trials, the subject was told how many Xs comprised the total matrix, $4,9,25$, or 49 , respectively. The subject was asked to estimate the number of Xs he or she observed. 
Table 1

Display Times

\begin{tabular}{ccccc}
\hline $\begin{array}{c}\text { Matrix } \\
\text { Size }\end{array}$ & $\begin{array}{c}\text { Number of Xs } \\
\text { Displayed }\end{array}$ & $\begin{array}{c}\text { On } \\
\text { Time }\end{array}$ & $\begin{array}{c}\text { Off } \\
\text { Time }\end{array}$ & $\begin{array}{c}\text { Total Display } \\
\text { Time }\end{array}$ \\
\hline 2 by 2 & 4 & 20 & 20 & 160 \\
3 by 3 & 9 & 10 & 10 & 180 \\
5 by 5 & 25 & 10 & 0 & 250 \\
7 by 7 & 49 & 10 & 0 & 490 \\
\hline
\end{tabular}

\section{RESULTS}

Table 2 gives the estimated number of Xs observed, averaged over the five subjects for the random displays. (For the nonrandom displays, in every case all subjects reported seeing all of the Xs displayed.) When the number of Xs actually displayed was 25 or 49 , the subject estimated seeing only about $60 \%$ of the letters. One point of view is that it should not be too surprising that the estimated number is much less than the actual number presented. As the number of displayed Xs becomes large ( 25 or 49 ), it becomes more difficult to estimate the larger number of Xs in such a short time. To counter this explanation, it is important to recall that each subject was told the total number of Xs in the matrix. For example, before the set of trials with 7 by 7 arrays, the subject was told that 49 Xs would be displayed in each trial. Further, the 10 nonrandom trials interspersed with the random ones helped to reinforce the structure of the matrix as consisting of 49 Xs. Even with such assurances, the subject consistently estimated fewer Xs than the actual number displayed.

In informal comments after the experiments, subjects consistently mentioned "holes" or empty spaces in the matrices. Such comments are consistent with those of subjects who have seen the linear lists under conditions of sequential blanking.

Table 2 clearly shows that with nine or more characters, significantly fewer Xs were reported as having been seen, compared with the actual number displayed. It was of interest whether a subject showed any pattern within a set of trials. For example, if there were some practice effect at work, the subject might report seeing more Xs as the trials go on. A t test was conducted for each of the four matrix sizes, comparing the average number reported for the subjects' first 10

Table 2

Estimated Number of Xs Seen

\begin{tabular}{cccc}
\hline & & \multicolumn{2}{c}{ Estimated Number of Xs } \\
\cline { 3 - 4 } $\begin{array}{c}\text { Matrix } \\
\text { Size }\end{array}$ & $\begin{array}{c}\text { Number } \\
\text { of Xs } \\
\text { Displayed }\end{array}$ & $\begin{array}{c}\text { Mean Over } \\
\text { All Sub- } \\
\text { jects }\end{array}$ & $\begin{array}{c}\text { Percent of } \\
\text { Actual Number } \\
\text { Displayed }\end{array}$ \\
\hline 2 by 2 & 4 & 3.65 & 91.25 \\
3 by 3 & 9 & 6.64 & 73.77 \\
5 by 5 & 25 & 14.56 & 58.24 \\
7 by 7 & 49 & 30.46 & 62.16 \\
\hline
\end{tabular}

random displays with the average for the last 10 random displays. The differences were not significant $(p>.25)$, thus suggesting that the responses were fairly consistent within the trials.

\section{DISCUSSION}

The sequential blanking effect has been chiefly associated with sequences of characters arranged in a horizontal line. Only two earlier results appear to deal with stimuli arranged vertically on the screen. In Mayzner and Tresselt (1970), one experiment used five point sources of light with a timing and ordering that produced the sequential blanking effect, blanking two of the five points. Moving the location of the two points that were blanked up to $.1 \mathrm{in}$. above or below the horizontal line of three blanking points did not interefere with sequential blanking. When the blanked points were moved more than $.1 \mathrm{in}$. above or below the line, the effect weakened. In the same experiment, Mayzner and Tresselt reported sequential blanking effects with polygons and a Necker cube. Both results show that the phenomenon is not restricted to linear arrangements of characters.

Two conditions must be fulfilled for sequential blanking to occur: (1) The inputs must be in close spatial proximity, and (2) the inputs must be separated in time by about $.1 \mathrm{sec}$. Both conditions are satisfied in the present study. With a random order of presentation used here, the spatiotemporal differences are enormously complex.

It is noteworthy that none of the percentage figures in Table 2 falls below 50\%. Explanations of sequential blanking usually center on the interaction between later arriving inputs and the partially processed earlier inputs. Under ideal conditions with the linear array, half of the inputs are blanked. With the random displays used in this experiment, some of the sequencing is not conducive to sequential blanking and somewhat less than half of the characters are blanked.

Continuing this line of thought would suggest further experimentation with matrix input. However, instead of random displays, one might design particular timing and ordering combinations to seek the maximum (or minimum) number of blanked characters for the same size matrix. Such results may increase our knowledge about excitatory and inhibitory field interactions.

In summary, the results indicate that there is a strong sequential blanking effect from such matrix displays. Experimenting with such complex two-dimensional input may be very useful in helping to expose the mechanisms that are at work in the processing of visual information.

\section{REFERENCES}

MAYzNer, M. S. The research potential of a computer-based cathode-ray tube display system. Behavior Research Methods \& Instrumentation, 1968, 1, 41-43.

MayzNER, M. S. Studies in visual information processing in man. In R. Solso (Ed.), Information processing and cognition. Hillsdale, N.J: Erlbaum, 1975.

Mayzner, M. S., \& Tresselt, M. E. Visual information processing with sequential inputs: A general model for sequential blanking, displacement, and overprinting phenomena. Annals of the New York Academy of Sciences, 1970, 169, 599-618. 